\title{
Finding the optimal treatment sequence in metastatic castration- resistant prostate cancer-a narrative review
}

\author{
Corinne Maurice Dror ${ }^{1}$, Kim N. Chi ${ }^{1,2}$, Daniel J. Khalaf ${ }^{1}$ \\ ${ }^{1}$ BC Cancer Vancouver, Vancouver, British Columbia, Canada; ${ }^{2}$ Vancouver Prostate Centre, Department of Urologic Sciences, University of British \\ Columbia, Vancouver, British Columbia, Canada \\ Contributions: (I) Conception and design: All authors; (II) Administrative support: None; (III) Provision of study materials or patients: None; (IV) \\ Collection and assembly of data: All authors; (V) Data analysis and interpretation: All authors; (VI) Manuscript writing: All authors; (VII) Final \\ approval of manuscript: All authors. \\ Correspondence to: Dr. Daniel J. Khalaf. Department of Medical Oncology, BC Cancer, Vancouver, 600 West 10th Avenue, Vancouver, British \\ Columbia, Canada. Email: daniel.khalaf@bccancer.bc.ca.
}

\begin{abstract}
Over the last two decades, there has been significant progress in the treatment of metastatic prostate cancer. Multiple treatments with diverse mechanisms of action have improved clinical outcomes for patients with metastatic castration-resistant prostate cancer (mCRPC) including taxane chemotherapy, immunotherapy, potent androgen receptor pathway inhibitors (ARPI), and radiopharmaceuticals (radium-223). As these treatments have entered standard clinical practise, clinicians have been challenged on how to optimally select and sequence them as the landmark studies establishing their efficacy had control arms with placebo or minimally effective therapy and there is a paucity of prospective trials examining treatment sequencing. More recently, the situation has been further complicated as the earlier up-front use of docetaxel and ARPI with standard gonadal testosterone inhibition has been shown to impart substantial improvements in disease control and survival for patients with castration sensitive prostate cancer. As new therapies enter into clinical practise such as the inhibitors of Poly (ADP-Ribose) Polymerase and Prostate Specific Membrane Antigen (PSMA)-targeted therapy, how to optimally select and sequence available treatments will be a continued dilemma in the absence of validated predictive biomarkers. This review will summarize the literature supporting the use of each active agent in MCRPC. We will propose a framework which will guide the selection of appropriate agents based on prior therapies, disease characteristics and biomarkers.
\end{abstract}

Keywords: Metastatic castration resistant prostate cancer; treatment; androgen receptor pathway inhibitors; taxanes; novel drugs; biomarkers

Submitted Oct 16, 2020. Accepted for publication Jan 29, 2021.

doi: $10.21037 /$ tau-20-1341

View this article at: http://dx.doi.org/10.21037/tau-20-1341

\section{Introduction}

Prostate cancer is the most common deadly malignancy in men and resulted in an estimated 33,330 deaths for males in the United States and 78,800 deaths in the European Union in $2020(1,2)$. Gonadal testosterone ablation was the earliest identified treatment for metastatic prostate cancer with proven efficacy. Its suppressive effect on metastatic prostate cancer was first demonstrated in 1941 by Huggins et al. (3), and suppression of gonadal androgen synthesis, either pharmacologically [luteinizing hormone releasing hormone (LHRH) antagonists or agonists, also referred to as androgen depravation therapy (ADT)] or surgically remains the backbone of treatment for prostate cancer in the metastatic state to this day $(4,5)$. Despite suppressed testosterone levels, prostate cancer will progress to a disease state, termed castration-resistant prostate cancer (CRPC) (6).

Encouragingly, the number of active treatment options for mCRPC has expanded considerably in recent years (Table 1). 
Docetaxel, a microtubule-stabilizing taxane chemotherapy, was the first drug to show survival benefit in the treatment of mCRPC (10). In addition to docetaxel's direct effect on microtubule dynamics, it may also act, in part, by inhibiting androgen receptor (AR) trafficking to the nucleus. In fact, activation of the AR pathway is a hallmark of prostate cancer and is the central driver of progression, even in the castration-resistant state, in the majority of patients (18). The AR pathway inhibitors (ARPIs) are a class of oral agents which include the CYP 17A1 inhibitor abiraterone acetate, administered with prednisone (AAP), which inhibits extragonadal androgen synthesis and the direct AR inhibitors enzalutamide, apalutamide and darolutamide. This class of agents is among the most effective and well tolerated for CRPC (7-9,19-22). Cabazitaxel, is a taxane chemotherapy with proven activity in patients who have progressed both on ARPI as well as docetaxel with a demonstrated survival benefit both after one or two lines of therapy (13). Lastly, the alpha-particle emitting radiopharmaceutical Radium-223, also prolongs survival in patients with predominantly bony metastases (14). All of the aforementioned drugs have become standard of care in the treatment of mCRPC, although the best sequence in which they should be administered is unknown.

Furthermore, a number of these drugs are now approved for the treatment of up-front castration-sensitive disease, with ADT. Indeed, consecutive phase III randomized placebo-controlled trials of docetaxel $(21,22)$ and AAP $(23,24)$ combined with ADT demonstrated a substantial delay in disease progression and improvement in overall survival, compared with upfront ADT alone. Recent phase III randomized trials of enzalutamide $(25,26)$ and apalutamide (27), have shown these agents to be similarly efficacious for metastatic castration-sensitive disease. This intensification of treatment earlier in the disease trajectory has undoubtedly been an important step forward, however, secondarily, the effectiveness of downstream therapy is diminished due to cross-resistance between agents and, potentially, development of more aggressive and treatment refractory disease phenotype upon progression to mCRPC.

Encouragingly, several novel agents for the treatment of $\mathrm{mCRPC}$ are anticipated to enter clinical practice in the near future. Notably, the Poly (ADP-Ribose) Polymerase (PARP) inhibitors have promising activity in tumours with genomic defects affecting double-stranded DNA repair by homologous-recombination, with olaparib (16) and rucaparib (28) now granted FDA approval and niraparib (29) which has been granted breakthrough therapy designation.
In addition, ${ }^{177}$ Lutetium-PSMA-617 a Prostate Specific Membrane Antigen (PSMA) targeting radioligand labeled with $\beta$-particle emitting Lutetium ${ }^{177}$ has shown promising activity in phase II trials (30).

This increase in the number of active agents poses a therapeutic dilemma, given the little data available to help select among the multiple possible treatment sequences. Indeed early landmark trials compared agents to now obsolete treatments such as mitoxantrone or placebo, and the majority of these proven agents have not been compared to one another in a randomized trial. A small numbers of trials have directly investigated treatments sequences and retrospective studies have provided insight into the degree of cross-resistance between treatments. Therapeutic choices are therefore guided by the side effect profile of the various agents, patient characteristics, potential biomarkers and individual clinician experience. In this review, the prospective evidence which supports current management of mCRPC will be summarized and the best evidence guiding the sequential use of therapies will be provided. Future promising agents as well as the emerging use of genomic biomarkers will also be described. We present the following article in accordance with the Narrative Review reporting checklist (available at http://dx.doi.org/10.21037/ tau-20-1341).

\section{Methods}

Literature was reviewed using PubMed and the following keywords were searched: metastatic castrate resistant prostate cancer, treatment and sequence/ing. A focus was put on publication within the last 20 years (2000 to 2020), however, key work published prior to this time frame was included. The articles were selected according to their design with a preference to randomized control trials, their relevance to this review and language (English). The references from all the articles found were searched for further relevant literature.

\section{Sequential use of ARPIs}

The ARPIs as well as the taxane chemotherapy docetaxel are mainstays of first line treatment for mCRPC. However, ARPIs are favored over docetaxel for first-line treatment based on safety and side-effect profile with docetaxel typically relegated to the second line. Furthermore, many patients may not be eligible for docetaxel due to comorbidities or frailty; hence a strategy of treating 


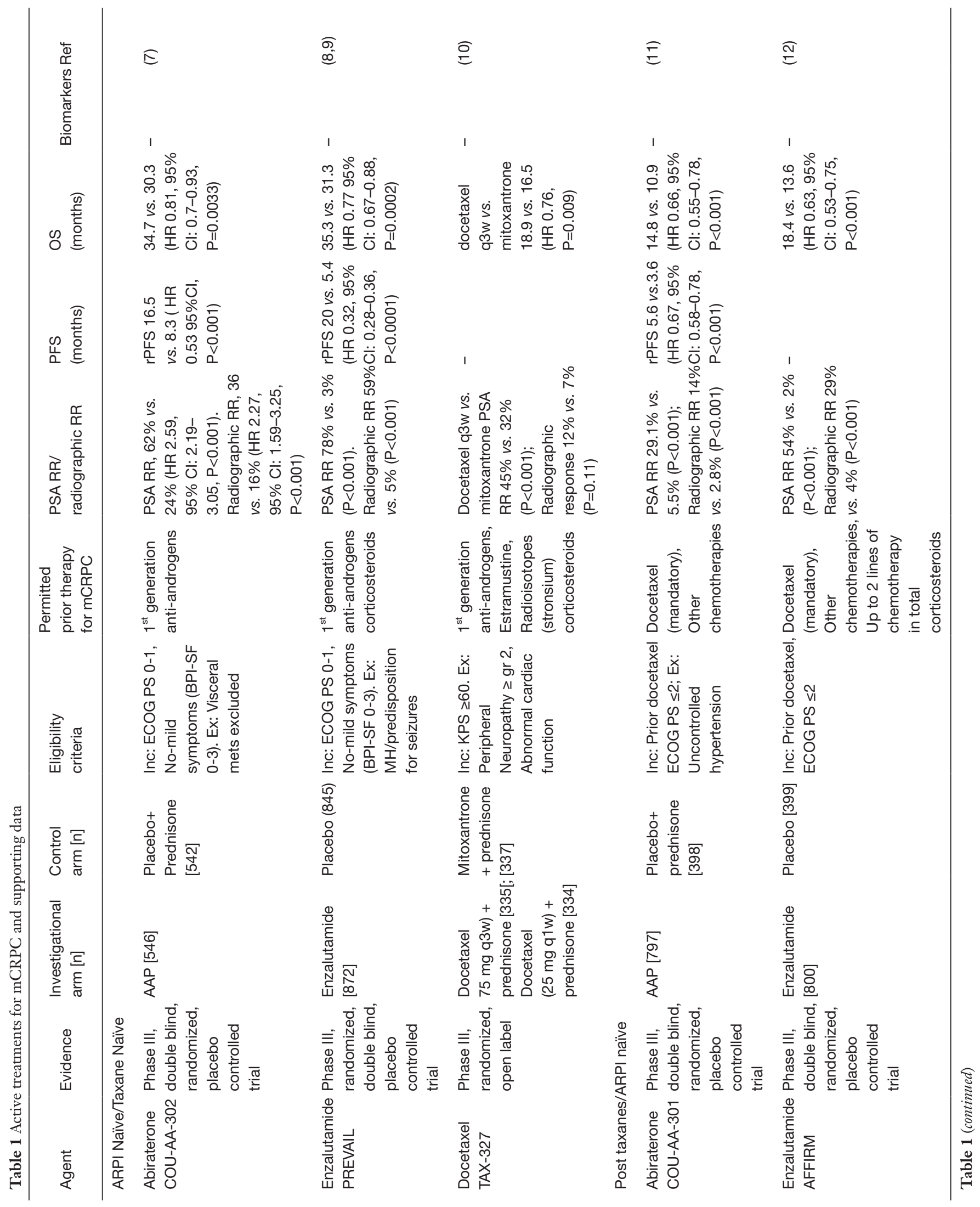




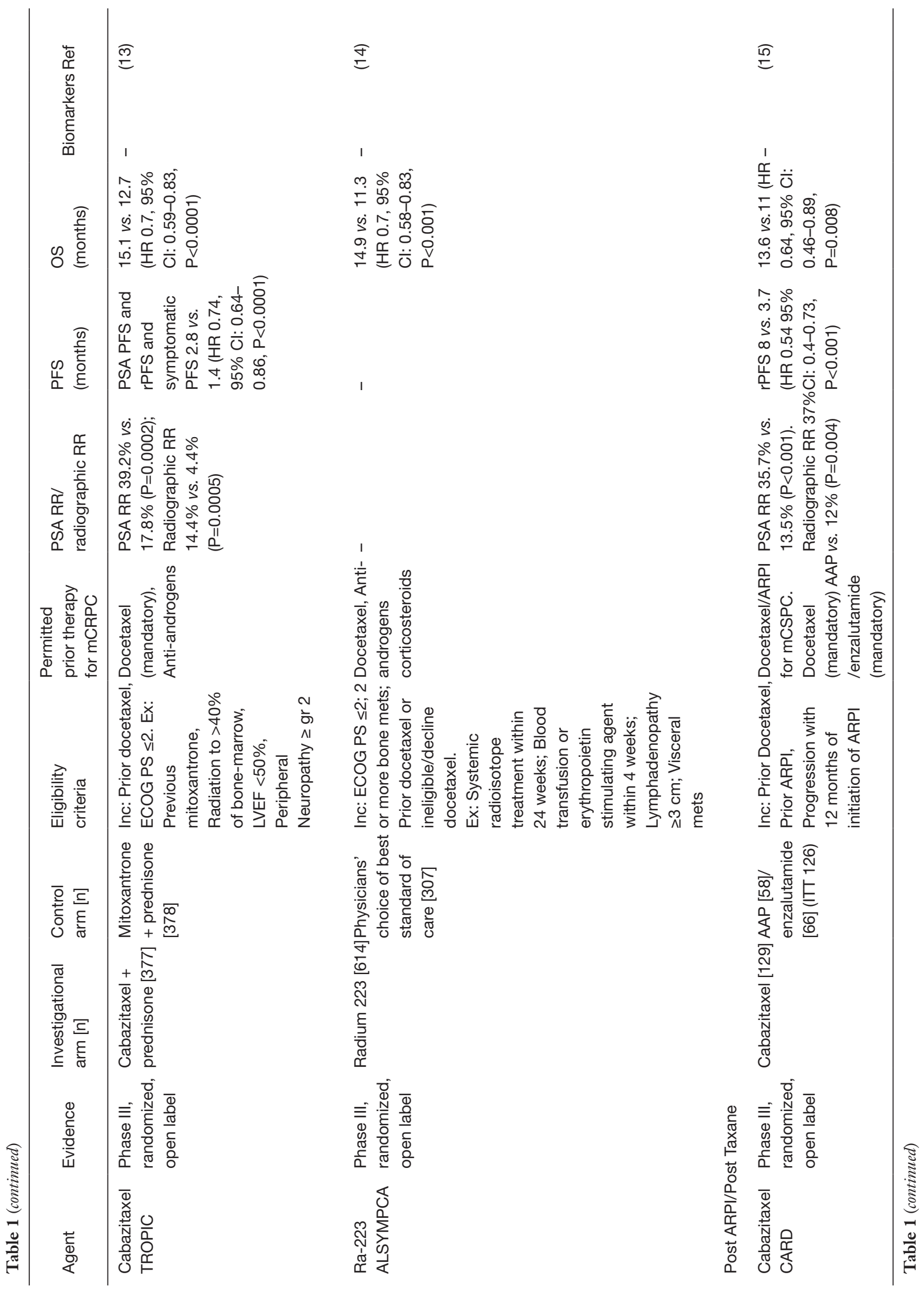


Translational Andrology and Urology, Vol 10, No 10 October 2021

3935

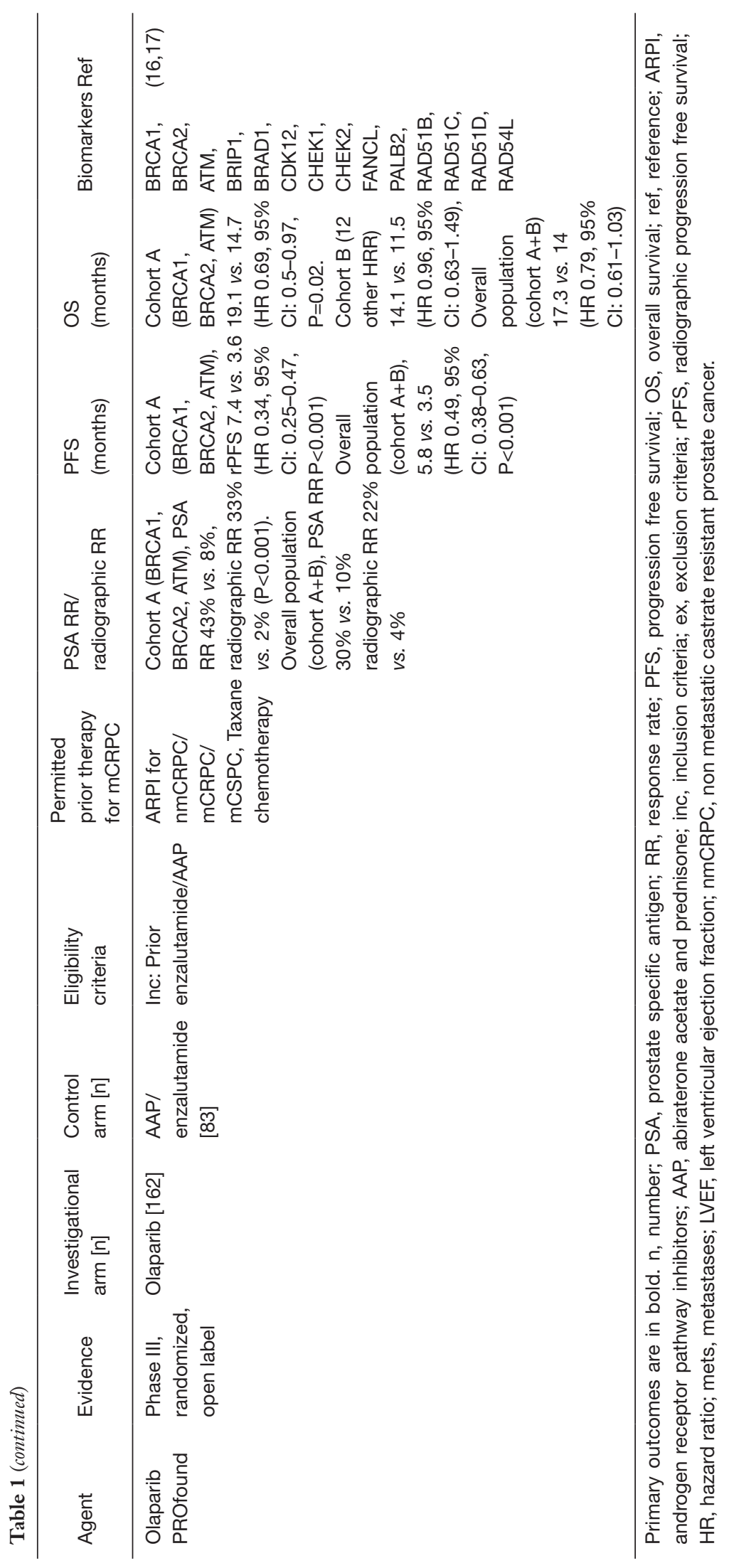

(C) Translational Andrology and Urology. All rights reserved.

Transl Androl Ural 2021;10(10):3931-3945 I http://dx.doi.org/10.21037/tau-20-1341 
with consecutive ARPIs has frequently been utilized in clinical practice and investigated retrospectively, but few prospective trials have been conducted (31-34). The optimal treatment sequencing of enzalutamide and AAP as first- and second-line agents was first prospectively studied in a phase II randomized trial. In this trial 202 patients were randomized to receive either AAP followed by enzalutamide at PSA progression vs. the opposite sequence (ABI-ENZA trial) (35). The key finding of the trial was that the treatment sequence of AAP followed by enzalutamide was associated with a superior time to second PSA progression compared with the opposite sequence (median 19.3 vs. 15.2 months, HR 0.66, 95\% CI: 0.45-0.97, $\mathrm{P}=0.036$ ). This was driven by improved efficacy of secondline enzalutamide compared with AAP including better time to PSA progression (median 3.5 vs. 1.7 months, HR 0.42, 95\% CI: $0.28-0.65, \mathrm{P}<0.0001)$ and better rate of confirmed $30 \%$ PSA decline (36\% vs. $4 \%, \mathrm{P}<0.0001)$. Patients with a time to progression of less than 3 months were less likely to benefit from a second ARPI. These results are supported by similar outcomes in smaller, retrospective or single arm design trials showing lack of significant activity of AAP post enzalutamide $(34,36,37)$ and modest activity of enzalutamide post AAP (38). Results of secondary endpoints of the study suggested that both enzalutamide and AAP are similarly efficacious in the first-line setting: although the PSA response rate was superior for enzalutamide $v s$. AAP in this setting (rate of confirmed 30\% PSA decline $82 \%$ vs. $68 \%$, respectively, $\mathrm{P}=0.023$ ), time to PSA progression was similar (median 11.2 vs. 10.2 months, HR 0.95, 95\% CI: 0.66-1.36). However, there was no overall survival advantage to one sequencing strategy over another (HR 0.79, 95\% CI: $0.54-1.16, \mathrm{P}=0.23)$. In clinical practice, other considerations that may guide the selection of first-line therapy include the side effect profile of each agent, patient comorbidities and age. AAP is associated with a risk of fluid retention, and caution is required for patients with a history of cardiovascular disease, particularly congestive heart failure (11). Enzalutamide is associated with a risk of seizures, in particular for patients with a history of intracranial lesions, or previous intracranial surgery, and may also cause excess fatigue as well mental status impairment (12). In the ABI-ENZA trial, healthrelated quality of life was shown to be inferior for men $\geq 75$ years treated with enzalutamide, but not for younger patients (39). This result is thought to reflect a higher burden of fatigue and cognitive effects associated with enzalutamide in the elderly patient population. In line with this hypothesis, a prospective study assessing patient reported outcomes in patients treated with AAP or enzalutamide, per investigator's choice, demonstrated fatigue and cognitive scores were inferior for enzalutamide $(40,41)$. Although selecting a sequence of ARPIs may be suitable for some patients, particularly those not suitable for treatment with chemotherapy, in the absence of a survival advantage or biomarkers predicting a response to a second APRI, alternating to another class of agents is preferable in most cases.

\section{Sequencing of ARPI and chemotherapy}

Due to the absence of direct comparative randomized data, the choice of initial therapy between ARPI or a taxane poses a challenge. In general terms, based on current practice guidelines (42), the majority of eligible patients will receive an ARPI and a taxane in either order. An important consideration is the diminished activity of agents in the second line, highlighting the importance of determining the ideal initial therapy. A degree of cross-resistance between docetaxel and ARPIs may exist due to a shared inhibitory effect on the AR (43-45). Retrospective clinical data indicates continued activity of docetaxel post-ARPIs with a PSA decline $\geq 50 \%$ of $26-35 \%$ (46-48) and phase III studies have shown a survival benefit of AAP and enzalutamide in the post-docetaxel setting $(11,12)$. Hence, this partial cross resistance should not preclude docetaxel in subsequent lines of treatment following ARPIs or the opposite sequencing. An important consideration is that the main phase III trials of AAP and enzalutamide accrued patients with absent to mild pain, inherently a better-prognosis population, and extrapolation of this data to higher risk patients should be done with caution $(7,11,12,49)$. Certain clinical factors, including poor response to prior ADT and visceral metastasis, are associated with worse outcomes on ARPI and prognostic indices have been developed which identify subgroups of patients with particularly poor prognosis when treated with AAP (50) or enzalutamide (51,52). Conversely, combination chemotherapy has shown high response rates in patients with a particular poor prognosis disease (53). Ultimately; it is important to define the optimal initial treatment choice in poor prognosis patients of which many will not reach next line of therapy. A recent randomized phase II cross-over trial (OZM-054) (54) compared a sequence of cabazitaxel and ARPIs (AAP or enzalutamide at investigator's discretion) $v s$. the opposite sequence in patients with poor prognosis mCRPC that were ARPI- 
naive. Poor prognosis was defined as liver metastases, early CRPC (<12 months from ADT start to castrate resistance), and/or presence of $\geq 4$ of 6 poor prognostic criteria (LDH $>$ upper limit of normal (ULN), ECOG PS 2, presence of liver metastases, albumin $\leq 4 \mathrm{~g} / \mathrm{dL}$, Alkaline phosphatase > ULN, time from start of ADT to initiation of treatment for mCRPC $<36$ months) (50). The primary outcome of the trial was clinical benefit rate (CBR) defined as PSA decline $\geq 50 \%$, measurable disease response and/or stable disease $>12$ weeks. Study accrual was terminated early in part due to slow enrollment. Ninety-five patients were enrolled and of these, approximately 50\% received prior docetaxel, for either mCSPC (29\% and 24\% in the cabazitaxel and ARPI cohorts, respectively) or mCRPC (24\% and 30\%). CBR in first line treatment was greater in the cabazitaxel first arm than the ARPI arm (88.4\% vs. $70 \% ; \mathrm{P}=0.043)$. All other measures including CBR in second line did not differ significantly. Of note, median overall survival was numerically longer in the cabazitaxel first arm $(37$ months, 95\% CI: 18.9-NR) vs. the ARPIs (15.5 months, 95\% CI: 12.4-NR). Although this trial cannot definitively determine whether chemotherapy or ARPI should be used up-front, the results support the view that poor prognosis disease phenotypes remain chemotherapy-responsive and may be better managed with upfront chemotherapy.

The question of optimal treatment in the third line post-docetaxel, post-ARPI setting was addressed in the CARD trial (15). In this phase III, randomized, open label trial, patients who previously received both docetaxel and one ARPI and whose disease progressed during the initial 12 months of treatment with the ARPI, were randomized in a $1: 1$ ratio to cabazitaxel at a dose of $25 \mathrm{mg} / \mathrm{m}^{2}$ every 3 weeks with standard supportive measures or ARPI not previously administered (patients who previously received AAP were randomized to receive enzalutamide and vice versa). All measures, including the primary outcome, median radiographic progression free survival ( $8 v s$. 3.7 months; HR 0.54, CI: 0.4-0.73, $\mathrm{P}<0.001)$, median overall survival (13.6 vs. 11 months HR 0.64, CI: 0.46-0.89, $\mathrm{P}=0.008)$, PSA decline $\geq 50 \%(37.5 \%$ vs. $13.5 \% ; \mathrm{P}<0.001)$ and confirmed pain response (45\% vs. $19.3 \%$ ) were superior in the cabazitaxel arm compared to the ARPI arm. In a post-hoc analysis, cabazitaxel maintained its superiority regardless of type of ARPI and the timing of treatment with ARPI in relation to docetaxel. Of note, the rate of serious adverse events was similar in both arms ( 39\%), however, adverse events leading to treatment discontinuation were more common in the cabazitaxel arm (19.8\% vs. $8.9 \%)$.
These results have established the superiority of cabazitaxel over a second ARPI. The ARPI arm may have performed poorly given that short lived response to first ARPI correlate with poor response to subsequent ARPI (55), and therefore results may not be generalizable to patients with long-term response to ARPI. Nevertheless, cabazitaxel is the first treatment to demonstrate a survival benefit in third line for mCRPC, an important finding which has solidified the role of cabazitaxel in this advanced setting.

\section{How does this data apply to agents that have moved to earlier disease setting?}

It is now standard practice to use either docetaxel, AAP, enzalutamide or apalutamide for the majority of patients in the setting of mCSPC. Furthermore, enzalutamide, apalutamide and darolutamide, novel AR inhibitors, are now indicated for use for patients who transition through a nonmetastatic castration-resistant disease state $(19,20,56)$. Thus, many patients will have been exposed to at least one ARPI or docetaxel at the emergence of mCRPC. Analyses of time to second progression (PFS2) from the phase III SPARTAN and TITAN trials of apalutamide + ADT $v s$. placebo + ADT demonstrated that the benefit of early treatment with ARPI extends beyond subsequent treatments, irrespective of whether taxane or ARPI is used at progression. $(19,27,57)$. However, these analyses did not directly compare the use of taxane $v$ s. second ARPI post apalutamide. In practice, it is assumed that cross-resistance between agents operates in the same manner when the first is received in the mCSPC setting or for mCRPC. However, prospective data are lacking, as to whether response to subsequent therapy is identical in these 2 settings, or may differ either mechanistically or in terms of rates of response. The considerations taken in determining subsequent treatment for patients who have progressed post docetaxel/ARPIs for $\mathrm{mCSPC}$ are similar to those taken in prescribing second line and beyond in the pre docetaxel/ARPIs for mCSPC era. For those patients who were treated with ARPIs in the mCSPC setting, concerns of cross-resistance should guide the clinicians to favor other classes of drugs. Similar considerations could be adopted for patients who received docetaxel in the mCSPC state, however, reintroduction of docetaxel in the mCRPC setting may also be an option. This approach was commonly utilized prior to emergence of alternative potent agents for mCRPC and limited, primarily retrospective data demonstrated a PSA decline $\geq 50 \%$ in $25-50 \%$ of patients re-introduced to docetaxel 
(58-60). These studies showed a correlation between docetaxel free interval until progression and efficacy of docetaxel re-introduction (61). A retrospective analysis (62) of the GETUG-AFU15 phase 3 trial comparing docetaxel and $\mathrm{ADT}$ to $\mathrm{ADT}$ alone for $\mathrm{mCSPC}$ examined the efficacy of docetaxel re-introduction for mCRPC following docetaxel treatment in the mCSPC setting. In this trial, patients who received docetaxel for mCSPC showed a more modest response when re-introduced to docetaxel in the mCRPC setting in the first line with a PSA decline $\geq 50 \%$ of $20 \%$ (4/20; $95 \%$ CI: 6-44). Additional prospective data is required to determine the utility of docetaxel reintroduction as well as the appropriate sequencing of treatment after exposure to ARPIs in the mCSPC setting. Until such data is available, clinicians may be cautiously guided by sequencing trials established in the mCRPC setting as well as patient and physician preferences.

\section{New therapies in mCRPC}

The landscape of treatment for mCRPC is continuously evolving with a number of trials examining the efficacy of new classes of drugs ongoing and recently reported. The discovery in the past decade that $20-30 \%$ of patients with mCRPC harbour underlying genomic defect in genes involved in DNA damage repair (DDR) $(63,64)$, prompted the investigation of PARP inhibitors as a novel therapeutic option. Phase I/II $(65,66)$ studies demonstrated that the identification of a germline or somatic defect in homologous recombination repair (HRR)genes, a subset of genes involved in DDR, particularly BRCA2 alterations, are predictive for a response to the PARP inhibitor, olaparib. The PROfound (16), a phase III, randomized, open label study enrolled patients with mCRPC with a somatic or germline defect in genes involved in HRR with prior exposure to ARPIs, enzalutamide or AAP. Patients could have also received prior taxane chemotherapy. Patients in cohort $\mathrm{A}$ of the trial, harboured a $B R C A 1, B R C A 2$ or $A T M$ defect, while those in cohort $\mathrm{B}$ had one of 12 other HRR defects (BRIP1, BARD1, CDK12, CHEK1, CHEK2, FANCL, PALB2, PPP2R2A, RAD51B, RAD51C, RAD51D, $R A D 54 L$ ). Both cohorts were randomized in a 2:1 ratio to receive either olaparib or an ARPI (AAP or enzalutamide per investigator's choice). Cross-over at progression was permitted. Radiographic progression-free survival, the primary outcome of the trial, was superior for olaparib in cohort A (median 7.4 vs. 3.6 months, HR 0.34; 95\% CI: 0.25 to $0.47 ; \mathrm{P}<0.001$ ) and in the overall study population (median
5.8 vs. 3.5 months, HR $0.49 ; 95 \%$ CI: 0.38 to $0.63 ; \mathrm{P}<0.001$ ). Grade 3 or higher adverse events were more common in the olaparib arm (51\% vs. 38\%) The most common of which were anemia (39\%), nausea (36\%) and fatigue/asthenia (32\%). In a final analysis, overall survival in cohort A, a key secondary end point, was significantly longer in the olaparib arm than in the control arm (19.1 vs. 14.7 months, HR 0.69; 95\% CI: 0.5-0.97; $\mathrm{P}=0.02)$. Cohort A derived the greatest survival benefit from olaparib, particularly patients with BRCA2 alterations, while there was no significant survival benefit in cohort $\mathrm{B}$ or the entire cohort, even after adjusting for crossover (17). This distinction between cohorts could be driven by gene specific response, however, the study was not powered to test such hypothesis. The results of this study established the efficacy of olaparib in the treatment of patients with mCRPC who harbor germline or somatic mutations in HRR, particularly for BRCA1, BRCA2 and ATM alterations. However, the trial could not determine the efficacy of olaparib in lower prevalence HRR genes which were grouped in cohort B, and further data will be required to elucidate the spectrum of alterations conferring sensitivity to PARP inhibitors. Interestingly, patients in this trial who were initially randomized to the control arm and then crossed over to the olaparib arm had a shorter lived radiographic progression free survival on olaparib when compared to those randomised to the olaparib arm (4.8 vs. 7.6 months), suggesting that olaparib treatment at an earlier line of treatment, in particular prior to a second line of ARPI, may fare better. In addition, patients with BRCA1/2 alterations who were taxane-naïve appeared to have greater survival benefit with olaparib (HR 0.3, 0.1-0.78) than those patients that were taxane-experienced (HR 0.64, 0.39-1.08). This suggests that olaparib should be preferentially used prior to taxane chemotherapy in this subgroup of patients rather than a second ARPI, irrespective of the sequence. The control arm of the PROFound trial also provides further evidence of the limited clinical activity of switching ARPI in patients already exposed to prior ARPI, as also demonstrated in the CARD and abi-enza trials. Further investigation is required to fully define the optimal sequencing of olaparib within the treatment paradigm of mCRPC harboring HRR mutations. Additional studies examining the efficacy of other PARP inhibitors in this late stage mCRPC setting (67-69) and the utility of PARP inhibitors in combination with other active agents in earlier lines of treatment are ongoing.

Another promising therapeutic is ${ }^{177} \mathrm{Lu}$-PSMA-617, a prostate-specific membrane antigen (PSMA)-targeting agent bound to beta-particle emitting radioisotope 
(Lutetium-177). This agent has demonstrated high level of activity and tolerable adverse event profile among mCRPC patients with ${ }^{6} \mathrm{Ga}$-PSMA-11 PET/CT avid tumors in nonrandomized and retrospective studies $(30,70,71)$. In a phase II, non-randomized clinical trial $57 \%$ of mCRPC patients treated with ${ }^{177} \mathrm{Lu}-\mathrm{PSMA}-617$ achieved a PSA decline $\geq 50 \%$ (30). TheraP (72), an ongoing phase II randomized trial [NCT03392428 (73)] comparing ${ }^{177}$ Lu-PSMA-617 to cabazitaxel in men with mCRPC who progressed after docetaxel, recently reported preliminary results. In this trial ${ }^{177} \mathrm{Lu}-\mathrm{PSMA}-617$ was given at a dose of 6-8GBq q6weeks for up to 6 cycles and cabazitaxel was prescribed at a dose of $20 \mathrm{mg} / \mathrm{m}^{2} \mathrm{q} 3$ weeks for up to 10 cycles. The majority of patient had more than 20 sites of disease $(78 \%)$ and had received prior treatment with ARPIs (91\%). PSA response, the primary outcome of the trial, defined as PSA decline $\geq 50 \%$, was superior in the ${ }^{177} \mathrm{Lu}-\mathrm{PSMA}-617$ arm (66\%; 95\% CI: $56-75 \%$ vs. $37 \%$; 95\% CI: $27-46 \%$; $\mathrm{P}<0.001)$. Furthermore, PSA-progression free survival was also improved in the ${ }^{177} \mathrm{Lu}-\mathrm{PSMA}-617$ arm (HR 0.63, 95\% CI: $0.45-0.88, \mathrm{P}=0.007)$. Decision regarding approval of this agent is pending more mature data including results from the VISION trial [NCT03511664 (74)], which randomized patients with mCRPC having previous received ARPI and docetaxel to receive either ${ }^{177} \mathrm{Lu}$-PSMA-617 or best supportive care. Defining the role of these new agents in the already crowded treatment scheme for MCRPC will be an important goal, which may be achieved through further comparative studies between agents, and by identification and validation predictive biomarkers.

\section{Biomarkers}

The sequencing trials detailed above provide some indication as to how to best approach treatment choice for mCRPC. However, the endeavor to prospectively define optimal sequencing of treatment for mCRPC by comparing all potential agents, at all lines of treatment, particularly, as new agents emerge in the continuously evolving treatment landscape of $\mathrm{mCRPC}$, is impractical. Predictive biomarkers offer a different approach to treatment tailoring by predicting response to different agents according to patients' specific characteristics. Such is the case for HRR mutations and treatment with PARP inhibitors as well as ${ }^{68}$ Ga-PSMA-11 PET/CT avidity and treatment with ${ }^{177} \mathrm{Lu}-$ PSMA-617 in mCRPC patients. Both HRR mutations and PET avidity serve as positive predictive marker for their respective treatments. Other potential predictive bio- markers are investigated in ongoing studies.

AR splice variant 7 (AR-V7) is one of the wellestablished resistance mechanisms to treatments targeting AR in mCRPC. This variant of AR lacks the ligand binding domain and is constitutively activated. AR-V7 may be found in approximately $10-30 \%$ of men with mCRPC. There are 2 validated methods to detect AR-V7 either via nuclear protein detection (Epic) or mRNA detection in circulating tumor cells (AdnaTest) (75). Studies comparing the response to ARPIs in AR-V7(+) and AR-V7(-) mCRPC patients suggest improved response among men with ARV7(-) mCRPC (76-78). In a study utilizing AR-V7 Epic nuclear protein detection Sharp et al. (77) demonstrated that AR-V7 is rarely expressed in primary prostate cancer. However, its prevalence increases dramatically following treatment with ADT, rising even further following treatment with abiraterone or enzalutamide, suggesting that the variant is developed under the selective pressure of $\mathrm{AR}$ inhibition. In this retrospective study, AR-V7 (+), docetaxel naïve patients were shown to have inferior PSA response (54\% vs. $100 \% ; \mathrm{P}=0.03)$ and overall survival (25.2 vs. 74.3 months, HR $0.23,0.07-0.79, \mathrm{P}=0.02)$ in response to ARPIs when compared to AR-V7(-) docetaxel naïve patients, this is in contrast to the response to docetaxel in which case no significant differences were seen between ARV7(+) and AR-V7(-) patients. Similarly, in the PROPHECY trial (78), AR-V7(+) tumors were associated with a shorter overall survival and progression free survival in response to ARPIs, when compared to AR-V7(-) tumors. The whole of this data suggest a potential, yet limited, role for AR-V7 as a negative predictive biomarker for the response to ARPIs in view of its low prevalence in the pre-ARPI settings. Prospective AR-V7 biomarker driven randomized clinical trials are needed to define the role of this AR splicing variant isoform in treatment selection.

Plasma circulating tumour DNA (ctDNA) has been extensively researched as a minimally invasive tool to profile mCRPC. Data from a number of groups has shown that ctDNA in mCRPC is abundant and detectable in a majority of patients, it correlates with overall tumour burden and provides a more objectively quantifiable, homogenous marker than disease burden (79-81). Within the aforementioned ABI-ENZA trial, patients provided serum for ctDNA analysis, allowing correlation of findings with outcomes, and determination of putative prognostic biomarkers in the setting of 1st line ARPI for mCRPC (79). In this analysis, the proportion of ctDNA of the plasma circulating free DNA (ctDNA\%) was examined 
and the cohort was subdivided into high ctDNA\% (>30\%), low ctDNA\% (2-30\%) and undetectable ctDNA\% $(<2 \%)$. Higher $\mathrm{ctDNA} \%$ correlated with worse time to progression and OS, while a ctDNA $\%>30 \%$ (compared with undetectable ctDNA), correlated most strongly with overall survival, independently of other clinical prognostic factors (HR 12.92, 95\% CI: 5.68-29.4; $\mathrm{P}<0.001$ ). Similar findings were shown in the OZM-054 trial, detailed above (54). Of the 95 patients enrolled in the trial, 76 (80\%) had ctDNA\% $>2 \%$. The most significant finding of this trial was that High baseline ctDNA\% correlated with both a poorer progression free survival (HR 6.58, 95\% CI: 2.95-14.69, $\mathrm{P}<0.001$ ) and overall survival (HR 25.43, 95\% CI: 3.36-190.99, $\mathrm{P}=0.002$ ) when compared to undetectable ctDNA\%, as was low ctDNA\% when compared to undetectable ctDNA\% (HR 3.05, 95\% CI: 1.52-6.14, $\mathrm{P}=002$ and HR 14.04, 95\% CI: 1.89-104.1, $\mathrm{P}=0.01$, respectively). This was maintained in a multivariate analysis, demonstrating that $\mathrm{ctDNA} \%$ is prognostic, independent of known clinical prognostic factors including the presence of visceral metastasis, elevated lactate dehydrogenase (LDH) and elevated alkaline phosphatase (ALP). A phase II trial (NCT04015622) (82) designed to elucidate the predictive role and clinical utility of ctDNA\% will compare ctDNA\% guided treatment to clinician choice of treatment. Patients randomized to the biomarker guided arm with ctDNA\% $>2$ will receive docetaxel, while patients with ctDNA\% $<2$ will receive enzalutamide. Patients in the clinician guided arm will receive enzalutamide or docetaxel according to clinician choice. Additional findings of both the ABI-ENZA and OZM-054 studies were that somatic and/or germline gene alterations in TP53, BRCA2, ATM, RB1, AR and the PI3K pathway also correlated with worse outcomes on ARPI. Numerous studies have now demonstrated the deleterious prognostic impact of tumour suppressor loss, particularly TP53 and RB1, both in the setting of metastatic castration-sensitive disease and mCRPC (83-86). Although BRCA2 mutations have been associated with a higher rate of disease progression and metastasis for localized disease, some studies have shown conflicting results with regards to the prognostic and predictive impact of BRCA2 in mCRPC (87-90). Collectively, these findings suggest that ctDNA\% as well as detection of germline and somatic genomic alterations may provide prognostic, and possibly predictive data. This concept requires prospective evaluation.

\section{Conclusions}

In the past decade the treatment paradigm of advanced prostate cancer has been substantially modified, initially by introduction of new active agents in the treatment of this disease state, followed by shift of treatment with docetaxel and ARPIs to the mCSPC setting and, more recently, the addition of 'targeted therapy' with olaparib for patients harboring HRR aberrations. This progress in the treatment of metastatic prostate cancer has had a positive impact on disease control and patients' survival. However, while all of the aforementioned therapies have well established efficacy, data is lacking regarding the optimal sequencing of these drugs. In the future, new drugs such as ${ }^{177} \mathrm{Lu}$-PSMA-617, may continue to re-define standard of care in the mCRPC setting and will further challenge clinical decision making around selecting and sequencing therapies.

Recent head-to-head comparator studies have shed some light on the optimal sequencing of AAP, enzalutamide and taxane chemotherapy in the first, second and third line of treatment. The results of the ABI-ENZA trial indicate that the sequencing of AAP followed by enzalutamide should be favoured over the opposite sequencing due to superior second line efficacy of the former sequence. In the CARD trial cabazitaxel was superior to ARPI among those patients with short lived response to ARPI in prior line and had been treated with prior docetaxel. Currently, this data along with considerations of the side effect profile of the various agents, patient characteristics and clinician's and patient's preferences provide limited guidance for clinician in treatment decision. Furthermore, enrollment in a clinical trial, if eligible, should always be considered.

As the treatment landscape for advanced prostate cancer continues to evolve, comparator trials aimed at defining the optimal sequence of agents will prove impractical. Alternatively, biomarkers offer a practical potential method to guide treatment choice, particularly with the advent in ctDNA analysis. Somatic and germline mutations have been shown to be a predictive biomarker for the response to treatment with PARP inhibitors in men with mCRPC. ARV7 status, AR gene amplification and aberrations, TP53 and RB1 defects and ctDNA\% have all been suggested to have a prognostic as well as possible predictive value. Nonetheless, standardization of these tests as well as prospective validation are required in order to determine their clinical utility. 


\section{Acknowledgments}

Funding: Daniel J. Khalaf received funding from the Prostate Cancer Foundation.

\section{Footnote}

Provenance and Peer Review: This article was commissioned by the Guest Editor (Tilman Todenhöfer) for the series "Management of Advanced Genitourinary Malignancies" published in Translational Andrology and Urology. The article has undergone external peer review.

Reporting Checklist: The authors have completed the Narrative Review reporting checklist. Available at http:// dx.doi.org/10.21037/tau-20-1341

Conflicts of Interest: All authors have completed the ICMJE uniform disclosure form (available at http://dx.doi. org/10.21037/tau-20-1341). The series "Management of Advanced Genitourinary Malignancies" was commissioned by the editorial office without any funding or sponsorship. CMD reports personal fees from BMS, MSD, Novartis and Medison, outside the submitted work. KNC reports grants and personal fees from Astellas, Astra Zeneca, Janssen, Novartis, Roche and Sanofi, and personal fees from Constellation Pharma, Daiichi Sankyo, Merck, Pfizer and Point Biopharma, outside the submitted work. The authors have no other conflicts of interest to declare.

Ethical Statement: The authors are accountable for all aspects of the work in ensuring that questions related to the accuracy or integrity of any part of the work are appropriately investigated and resolved.

Open Access Statement: This is an Open Access article distributed in accordance with the Creative Commons Attribution-NonCommercial-NoDerivs 4.0 International License (CC BY-NC-ND 4.0), which permits the noncommercial replication and distribution of the article with the strict proviso that no changes or edits are made and the original work is properly cited (including links to both the formal publication through the relevant DOI and the license). See: https://creativecommons.org/licenses/by-nc-nd/4.0/.

\section{References}

1. Carioli G, Bertuccio P, Boffetta P, et al. European cancer mortality predictions for the year 2020 with a focus on prostate cancer. Ann Oncol 2020;31:650-8.

2. Siegel RL, Miller KD, Jemal A. Cancer statistics, 2020. CA Cancer J Clin 2020;70:7-30.

3. Huggins C, Stevens RE, Hodges CV. Studies on prostatic cancer: ii. the effects of castration on advanced carcinoma of the prostate gland. Arch Surg 1941;43:209-23.

4. Malone S, Shayegan B, Basappa NS, et al. Management algorithms for metastatic prostate cancer. Can Urol Assoc J 2019;13:50.

5. Gillessen S, Attard G, Beer TM, et al. Management of Patients with Advanced Prostate Cancer: Report of the Advanced Prostate Cancer Consensus Conference 2019. Eur Urol 2020;77:508-47.

6. Taylor CD, Elson P, Trump DL. Importance of continued testicular suppression in hormone-refractory prostate cancer. J Clin Oncol 1993;11:2167-72.

7. Ryan CJ, Smith MR, Fizazi K, et al. Abiraterone acetate plus prednisone versus placebo plus prednisone in chemotherapy-naive men with metastatic castrationresistant prostate cancer (COU-AA-302): Final overall survival analysis of a randomised, double-blind, placebocontrolled phase 3 study. Lancet Oncol 2015;16:152-60.

8. Beer TM, Armstrong AJ, Rathkopf DE, et al. Enzalutamide in metastatic prostate cancer before chemotherapy. N Engl J Med 2014;371:424-33.

9. Beer TM, Armstrong AJ, Rathkopf D, et al. Enzalutamide in Men with Chemotherapy-naïve Metastatic Castrationresistant Prostate Cancer: Extended Analysis of the Phase 3 PREVAIL Study. Eur Urol 2017;71:151-4.

10. Berthold DR, Pond GR, Soban F, et al. Docetaxel plus prednisone or mitoxantrone plus prednisone for advanced prostate cancer: Updated survival in the TAX 327 study. J Clin Oncol 2008;26:242-5.

11. de Bono JS, Logothetis CJ, Molina A, et al. Abiraterone and increased survival in metastatic prostate cancer. $\mathrm{N}$ Engl J Med 2011;364:1995-2005.

12. Scher HI, Fizazi K, Saad F, et al. Increased survival with enzalutamide in prostate cancer after chemotherapy. $\mathrm{N}$ Engl J Med 2012;367:1187-97.

13. de Bono JS, Oudard S, Ozguroglu M, et al. Prednisone plus cabazitaxel or mitoxantrone for metastatic castrationresistant prostate cancer progressing after docetaxel treatment: A randomised open-label trial. Lancet 2010;376:1147-54.

14. Parker C, Nilsson D Heinrich S, Helle SI, et al. Alpha emitter radium-223 and survival in metastatic prostate 
cancer. N Engl J Med 2013;369:213-23.

15. de Wit R, De Bono J, Sternberg CN, et al. Cabazitaxel versus abiraterone or enzalutamide in metastatic prostate cancer. N Engl J Med 2019;381:2506-18.

16. de Bono J, Mateo J, Fizazi K, et al. Olaparib for metastatic castration-resistant prostate cancer. N Engl J Med 2020;382:2091-102.

17. Hussain M, Mateo J, Fizazi K, et al. Survival with Olaparib in Metastatic Castration-Resistant Prostate Cancer. N Engl J Med 2020;383:2345-57.

18. Watson PA, Arora VK, Sawyers CL. Emerging mechanisms of resistance to androgen receptor inhibitors in prostate cancer. Nat Rev Cancer 2015;15:701-11.

19. Smith MR, Saad F, Chowdhury S, et al. Apalutamide treatment and metastasis-free survival in prostate cancer. N Engl J Med 2018;378:1408-18.

20. Fizazi K, Shore N, Tammela TL, et al. Darolutamide in Nonmetastatic, Castration-Resistant Prostate Cancer. N Engl J Med 2019;380:1235-46.

21. James ND, Sydes MR, Clarke NW, et al. Addition of docetaxel, zoledronic acid, or both to first-line longterm hormone therapy in prostate cancer (STAMPEDE): survival results from an adaptive, multiarm, multistage, platform randomised controlled trial. Lancet 2016;387:1163-77.

22. Sweeney CJ, Chen YH, Carducci M, et al. Chemohormonal Therapy in Metastatic Hormone-Sensitive Prostate Cancer. N Engl J Med 2015;373:737-46.

23. James ND, De Bono JS, Spears MR, et al. Abiraterone for prostate cancer not previously treated with hormone therapy. N Engl J Med 2017;377:338-51.

24. Fizazi K, Tran N, Fein L, et al. Abiraterone plus Prednisone in Metastatic, Castration-Sensitive Prostate Cancer. N Engl J Med 2017;377:352-60.

25. Davis ID, Martin AJ, Stockler MR, et al. Enzalutamide with standard first-line therapy in metastatic prostate cancer. N Engl J Med 2019;381:121-31.

26. Buonerba C, Ferro M, Dolce P, et al. Predictors of efficacy of androgen-receptor-axis-targeted therapies in patients with metastatic castration-sensitive prostate cancer: A systematic review and meta-analysis. Crit Rev Oncol Hematol 2020;151:102992.

27. Chi KN, Agarwal N, Bjartell A, et al. Apalutamide for metastatic, castration-sensitive prostate cancer. N Engl J Med 2019;381:13-24.

28. Abida W, Patnaik A, Campbell D, et al. Rucaparib in Men With Metastatic Castration-Resistant Prostate Cancer Harboring a BRCA1 or BRCA2 Gene Alteration. J Clin
Oncol 2020;38:3763-72.

29. Smith MR, Sandhu SK, Kelly WK, et al. Pre-specified interim analysis of GALAHAD: A phase II study of niraparib in patients (pts) with metastatic castrationresistant prostate cancer (mCRPC) and biallelic DNArepair gene defects (DRD). Ann Oncol 2019;30:v884-5.

30. Hofman MS, Violet J, Hicks RJ, et al. [177 Lu]-PSMA-617 radionuclide treatment in patients with metastatic castration-resistant prostate cancer (LuPSMA trial): a single-centre, single-arm, phase 2 study. Lancet Oncol 2018;19:825-33.

31. Khalaf DJ, Annala M, Taavitsainen S, et al. Optimal sequencing of enzalutamide and abiraterone acetate plus prednisone in metastatic castration-resistant prostate cancer: a multicentre, randomised, open-label, phase 2, crossover trial. Lancet Oncol 2019;20:1730-9.

32. Maines F, Caffo O, Veccia A, et al. Sequencing new agents after docetaxel in patients with metastatic castrationresistant prostate cancer. Crit Rev Oncol Hematol 2015;96:498-506.

33. Oh WK, Cheng WY, Miao R, et al. Real-world outcomes in patients with metastatic castration-resistant prostate cancer receiving second-line chemotherapy versus an alternative androgen receptor-targeted agent (ARTA) following early progression on a first-line ARTA in a US community oncology setting. Urol Oncol 2018;36:500.e1500.e9.

34. Attard G, Borre M, Gurney H, et al. Abiraterone alone or in combination with enzalutamide in metastatic castrationresistant prostate cancer with rising prostate-specific antigen during enzalutamide treatment. J Clin Oncol 2018;36:2639-46.

35. Khalaf DJ, Annala M, Taavitsainen S, et al. Optimal sequencing of enzalutamide and abiraterone acetate plus prednisone in metastatic castration-resistant prostate cancer: a multicentre, randomised, open-label, phase 2, crossover trial. Lancet Oncol 2019;20:1730-9.

36. Loriot $\mathrm{Y}$, Bianchini D, Ileana E, et al. Antitumour activity of abiraterone acetate against metastatic castrationresistant prostate cancer progressing after docetaxel and enzalutamide (MDV3100). Ann Oncol 2013;24:1807-12.

37. Noonan KL, North S, Bitting RL, et al. Clinical activity of abiraterone acetate in patients with metastatic castrationresistant prostate cancer progressing after enzalutamide. Ann Oncol 2013;24:1802-7.

38. de Bono JS, Chowdhury S, Feyerabend S, et al. Antitumour Activity and Safety of Enzalutamide in Patients with Metastatic Castration-resistant Prostate Cancer Previously 
Treated with Abiraterone Acetate Plus Prednisone for $\geq 24$ weeks in Europe. Eur Urol 2018;74:37-45.

39. Khalaf DJ, Sunderland K, Eigl BJ, et al. Health-related Quality of Life for Abiraterone Plus Prednisone Versus Enzalutamide in Patients with Metastatic Castrationresistant Prostate Cancer: Results from a Phase II Randomized Trial. Eur Urol 2019;75:940-7.

40. Moreira RB, Debiasi M, Francini E, et al. Differential side effects profile in patients with $\mathrm{mCRPC}$ treated with abiraterone or enzalutamide: A meta-analysis of randomized controlled trials. Oncotarget 2017;8:84572-8.

41. Shore ND, Saltzstein D, Sieber P, et al. Results of a Realworld Study of Enzalutamide and Abiraterone Acetate With Prednisone Tolerability (REAAcT). Clin Genitourin Cancer 2019;17:457-463.e6.

42. Freedman-Cass D, Shead DA, Schaeffer E, et al. NCCN Guidelines Panel Disclosures Continue \& Diagnostic/ Interventional radiology $\mathrm{P}$ Internal medicine $\uparrow$ Medical oncology $\neq$ Pathology $¥$ Patient advocate $§$ Radiotherapy/ Radiation oncology w Urology * Discussion Section Writing Committee NCCN Guidelines Version 2.2020 Prostate Cancer. 2020.

43. Zhu ML, Horbinski CM, Garzotto M, et al. Tubulintargeting chemotherapy impairs androgen receptor activity in prostate cancer. Cancer Res 2010;70:7992-8002.

44. van Soest RJ, Van Royen ME, De Morrée ES, et al. Crossresistance between taxanes and new hormonal agents abiraterone and enzalutamide may affect drug sequence choices in metastatic castration-resistant prostate cancer. Eur J Cancer 2013;49:3821-30.

45. Darshan MS, Loftus MS, Thadani-Mulero M, et al. Taxane-induced blockade to nuclear accumulation of the androgen receptor predicts clinical responses in metastatic prostate cancer. Cancer Res 2011;71:6019-29.

46. Mezynski J, Pezaro C, Bianchini D, et al. Antitumour activity of docetaxel following treatment with the CYP17A1 inhibitor abiraterone: Clinical evidence for cross-resistance? Ann Oncol 2012;23:2943-7.

47. Azad AA, Leibowitz-Amit R, Eigl BJ, et al. A retrospective, Canadian multi-center study examining the impact of prior response to abiraterone acetate on efficacy of docetaxel in metastatic castration-resistant prostate cancer. Prostate 2014;74:1544-50.

48. Chi K, Hotte SJ, Joshua AM, et al. Treatment of mCRPC in the AR-axis-targeted therapy-resistant state. Ann Oncol 2015;26:2044-56.

49. Beer TM, Armstrong AJ, Rathkopf D, et al. Enzalutamide in Men with Chemotherapy-naïve Metastatic Castration- resistant Prostate Cancer: Extended Analysis of the Phase 3 PREVAIL Study. Eur Urol 2017;71:151-4.

50. Chi KN, Kheoh T, Ryan CJ, et al. A prognostic index model for predicting overall survival in patients with metastatic castration-resistant prostate cancer treated with abiraterone acetate after docetaxel. Ann Oncol 2016;27:454-60.

51. Armstrong AJ, Lin P, Higano CS, et al. Development and validation of a prognostic model for overall survival in chemotherapy-naïve men with metastatic castrationresistant prostate cancer. Ann Oncol 2018;29:2200-7.

52. Armstrong AJ, Lin P, Tombal B, et al. Five-year Survival Prediction and Safety Outcomes with Enzalutamide in Men with Chemotherapy-naïve Metastatic Castrationresistant Prostate Cancer from the PREVAIL Trial. Eur Urol 2020;78:347-57.

53. Aparicio AM, Harzstark AL, Corn PG, et al. PlatinumBased Chemotherapy for Variant Castrate-Resistant Prostate Cancer. Clin Cancer Res 2013;19:3621-30.

54. Chi KN, Taavitsainen S, Iqbal N, et al. Updated results from a randomized phase II study of cabazitaxel (CAB) versus abiraterone $(\mathrm{ABI})$ or enzalutamide $(\mathrm{ENZ})$ in poor prognosis metastatic CRPC. J Clin Oncol 2019;37:5003.

55. Nadal R, Tsai HL, Sinibaldi VJ, et al. Prognostic factors for clinical outcomes in patients with metastatic castration resistant prostate cancer treated with sequential novel androgen receptor-directed therapies. Prostate 2016;76:512-20.

56. Hussain M, Fizazi K, Saad F, et al. Enzalutamide in men with nonmetastatic, castration-resistant prostate cancer. N Engl J Med 2018;378:2465-74.

57. Agarwal N, Chowdhury S, Bjartell A, et al. Time to second progression (PFS2) in patients (pts) from TITAN with metastatic castration-sensitive prostate cancer (mCSPC) by first subsequent therapy (hormonal $v s$. taxane). J Clin Oncol 2020;38:82.

58. Oudard S, Kramer G, Caffo O, et al. Docetaxel rechallenge after an initial good response in patients with metastatic castration-resistant prostate cancer. BJU Int 2015;115:744-52.

59. Eymard JC, Oudard S, Gravis G, et al. Docetaxel reintroduction in patients with metastatic castrationresistant docetaxel-sensitive prostate cancer: A retrospective multicentre study. BJU Int 2010;106:974-8.

60. Di Lorenzo G, Buonerba C, Faiella A, et al. Phase II study of docetaxel re-treatment in docetaxelpretreated castration-resistant prostate cancer. BJU Int 2011;107:234-9. 
61. Loriot Y, Massard C, Gross-Goupil M, et al. The interval from the last cycle of docetaxel-based chemotherapy to progression is associated with the efficacy of subsequent docetaxel in patients with prostate cancer. Eur J Cancer 2010;46:1770-2.

62. Lavaud P, Gravis G, Foulon S, et al. Anticancer Activity and Tolerance of Treatments Received Beyond Progression in Men Treated Upfront with Androgen Deprivation Therapy With or Without Docetaxel for Metastatic Castration-naïve Prostate Cancer in the GETUG-AFU 15 Phase 3 Trial. Eur Urol 2018;73:696-703.

63. Robinson D, Van Allen EM, Wu YM, et al. Integrative clinical genomics of advanced prostate cancer. Cell 2015;161:1215-28.

64. Abida W, Armenia J, Gopalan A, et al. Prospective Genomic Profiling of Prostate Cancer Across Disease States Reveals Germline and Somatic Alterations That May Affect Clinical Decision Making. JCO Precis Oncol 2017;2017:PO.17.00029.

65. Mateo J, Carreira S, Sandhu S, et al. DNA-Repair Defects and Olaparib in Metastatic Prostate Cancer. N Engl J Med 2015;373:1697-708.

66. Mateo J, Porta N, Bianchini D, et al. Olaparib in patients with metastatic castration-resistant prostate cancer with DNA repair gene aberrations (TOPARP-B): a multicentre, open-label, randomised, phase 2 trial. Lancet Oncol 2020;21:162-74.

67. A Study of Talazoparib in Men With DNA Repair Defects and Metastatic Castration-Resistant Prostate Cancer - Full Text View - ClinicalTrials.gov.

68. An Efficacy and Safety Study of Niraparib in Men With Metastatic Castration-Resistant Prostate Cancer and DNA-Repair Anomalies - Full Text View ClinicalTrials.gov.

69. A Study of Rucaparib in Patients With Metastatic Castration-resistant Prostate Cancer and Homologous Recombination Gene Deficiency - Full Text View ClinicalTrials.gov.

70. Rasul S, Hacker M, Kretschmer-Chott E, et al. Clinical outcome of standardized 177Lu-PSMA-617 therapy in metastatic prostate cancer patients receiving 7400 MBq every 4 weeks. Eur J Nucl Med Mol Imaging 2020;47:713-20.

71. Barber TW, Singh A, Kulkarni HR, et al. Clinical outcomes of $177 \mathrm{Lu}-\mathrm{PSMA}$ radioligand therapy in earlier and later phases of metastatic castration-resistant prostate cancer grouped by previous taxane chemotherapy. J Nucl Med 2019;60:955-62.
72. Hofman MS, Emmett L, Sandhu SK, et al. TheraP: A randomised phase II trial of 177 Lu-PSMA-617 (LuPSMA) theranostic versus cabazitaxel in metastatic castration resistant prostate cancer (mCRPC) progressing after docetaxel: Initial results (ANZUP protocol 1603). J Clin Oncol 2020;38:5500.

73. A Trial of $177 \mathrm{Lu}-P S M A 617$ Theranostic Versus Cabazitaxel in Progressive Metastatic Castration Resistant Prostate Cancer - Full Text View - ClinicalTrials.gov.

74. Study of 177Lu-PSMA-617 In Metastatic CastrateResistant Prostate Cancer - Full Text View ClinicalTrials.gov.

75. Brown LC, Lu C, Antonarakis ES, et al. Androgen receptor variant-driven prostate cancer II: advances in clinical investigation. Prostate Cancer Prostatic Dis 2020;23:367-80.

76. Antonarakis ES, Lu C, Wang H, et al. AR-V7 and resistance to enzalutamide and abiraterone in prostate cancer. N Engl J Med 2014;371:1028-38.

77. Sharp A, Coleman I, Yuan W, et al. Androgen receptor splice variant-7 expression emerges with castration resistance in prostate cancer. J Clin Invest 2019;129:192-208.

78. Armstrong AJ, Halabi S, Luo J, et al. Prospective multicenter validation of androgen receptor splice variant 7 and hormone therapy resistance in high-risk castrationresistant prostate cancer: The PROPHECY study. J Clin Oncol 2019;37:1120-9.

79. Annala M, Vandekerkhove G, Khalaf D, et al. Circulating tumor DNA genomics correlate with resistance to abiraterone and enzalutamide in prostate cancer. Cancer Discov 2018;8:444-57.

80. Alix-Panabières C, Pantel K. Clinical applications of circulating tumor cells and circulating tumor DNA as liquid biopsy. Cancer Discov 2016;6:479-91.

81. Azad AA, Volik S V., Wyatt AW, et al. Androgen receptor gene aberrations in circulating cell-free DNA: Biomarkers of therapeutic resistance in castration-resistant prostate cancer. Clin Cancer Res 2015;21:2315-24.

82. PROTRACT (PROstate Cancer TReatment Optimization Via Analysis of Circulating Tumour DNA) STUDY - Full Text View - ClinicalTrials.gov.

83. De Laere B, Oeyen S, Mayrhofer M, et al. TP53 outperforms other androgen receptor biomarkers to predict abiraterone or enzalutamide outcome in metastatic castration-resistant prostate cancer. Clin Cancer Res 2019;25:1766-73.

84. Aparicio AM, Shen L, Tapia ELN, et al. Combined Tumor 
Suppressor Defects Characterize Clinically Defined Aggressive Variant Prostate Cancers. Clin Cancer Res 2016;22:1520-30.

85. Hamid AA, Gray KP, Shaw G, et al. Compound Genomic Alterations of TP53, PTEN, and RB1 Tumor Suppressors in Localized and Metastatic Prostate Cancer. Eur Urol 2019;76:89-97.

86. Nyquist MD, Corella A, Coleman I, et al. Combined TP53 and RB1 Loss Promotes Prostate Cancer Resistance to a Spectrum of Therapeutics and Confers Vulnerability to Replication Stress. Cell Rep 2020;31:107669.

87. Castro E, Romero-Laorden N, Del Pozo A, et al. Prorepair-B: A prospective cohort study of the impact of germline DNA repair mutations on the outcomes of patients with metastatic castration-resistant prostate

Cite this article as: Maurice Dror C, Chi KN, Khalaf DJ. Finding the optimal treatment sequence in metastatic castration-resistant prostate cancer-a narrative review. Transl Androl Urol 2021;10(10):3931-3945. doi: 10.21037/tau-20-1341 cancer. J Clin Oncol 2019;37:490-503.

88. Hussain M, Daignault-Newton S, Twardowski PW, et al. Targeting androgen receptor and DNA repair in metastatic castration-resistant prostate cancer: Results from NCI 9012. J Clin Oncol 2018;36:991-9.

89. Mateo J, Cheng HH, Beltran H, et al. Clinical Outcome of Prostate Cancer Patients with Germline DNA Repair Mutations: Retrospective Analysis from an International Study. Eur Urol 2018;73:687-93.

90. Antonarakis ES, Lu C, Luber B, et al. Germline DNArepair Gene Mutations and Outcomes in Men with Metastatic Castration-resistant Prostate Cancer Receiving First-line Abiraterone and Enzalutamide. Eur Urol 2018;74:218-25. 